\title{
Improvements in calibration at silver or Copper point
}

\author{
J.P. Tavener* \\ Isothermal Technology Limited, Pine Grove, Southport, PR9 9AG, England
}

Received: 15 December 2009 / Accepted: 5 February 2010

\begin{abstract}
The main purpose of the paper is to describe the construction and performance of a new and novel Silver and Copper point system that combines a conventional ingot of silver or copper in a graphite crucible, with a specially designed heat dewar or heat siphon to form a single entity. Furthermore, to add above this entity a heated collar to exactly compensate for heat losses upwards and the stem conduction effects of the thermometer being calibrated. The overall construction contains no glass parts, is rugged, compact and transportable. Its performance is similar to large quartz clad cells in floor standing heat pipe apparatus at a fraction of the size and cost.
\end{abstract}

Keywords: Silver point system; Copper point system; CAFMET2010; article

\section{Introduction}

For contact thermometer calibration at the Copper Point, the Cell has traditionally been clad in quartz glass which is fragile and soft at this high temperature $\left(1084.62{ }^{\circ} \mathrm{C}\right)$.

It would be convenient if the Cell could be clad in metal. This article describes the construction of such a Cell with its Apparatus.

Part One [1] of this sequence described the development of a novel combination of Cell, Apparatus and Immersion Compensation for the ITS-90 Fixed Points of Indium, Tin, Zinc and Aluminium.

It had always been the intention to extend the range to Silver and Copper.

\section{Description}

One objective in extending the range to Silver and Copper is to eliminate the use of quartz glass that traditionally surrounds these cells. The glass is not only fragile and untransportable except by hand, but the glass is porous to ions such as Nickel, Chromium and Iron for example. The glass also softens and deforms especially at the Copper point and easily devitrifies.

Solutions already exist in Radiation Pyrometry for the Copper and Silver points which do not rely on glass.

Pyrometry has to use open cells where the graphite surrounding the metal is exposed so that it can be viewed by optical pyrometers.

This is achieved by flushing the cell assembly with $6 \mathrm{~N} 0$ pure Argon. The cell assembly is supported at these temperatures inside a sodium isothermal liner. A typical copper point furnace is illustrated in Supplementary Information for ITS-90 [2].

\footnotetext{
* Correspondence: info@isotech.co.uk
}

In pyrometry the cells are horizontal, whereas in Contact Thermometry the cells are mounted vertically.

\section{Construction of the siphonic cell}

The cell was housed in a heat syphon in the shape of a dewar, with extended inner tube as shown in Figure 1. A removable lid with re-entrant tube and Argon supply tube was fitted above the cell.

A heated metal collar was used to compensate the stem conduction of the test thermometer.

$6 \mathrm{~N} 0$ grade Argon was used to blanket the cell with a flow of $0.2 \mathrm{l} / \mathrm{m}$.

Argon is heavier than air and so quickly displaces the air in the cell.

To measure the temperature at the Silver and Copper point a new thermometer was designed and used.

The new thermometer differs from existing models in a number of novel ways.

Described elsewhere, it has an alumina rather than a quartz sheath to provide rigidity at these high temperatures.

Readings were made using a microK 100 with an external Tinsley $1 \Omega$ resistor held at $20{ }^{\circ} \mathrm{C}$.

\section{Results at the silver point}

The apparatus above was built - see Picture 1 -, and a series of melts and freezes were performed on the cell.

A typical Silver melt and freeze are shown in Figures 2 and 3 .

The three hours melt had an $80 \%$ slope of $3 \mathrm{mK}$ whilst the first $50 \%$ of the $3 \mathrm{~h}$ freeze was flat.

Melt and freeze were coincident. 


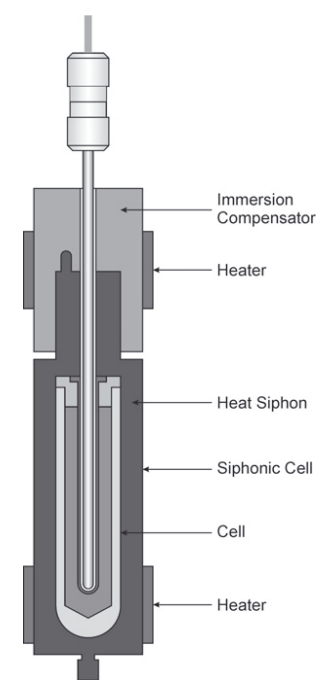

Fig. 1. Siphonic cell \& immersion compensator.

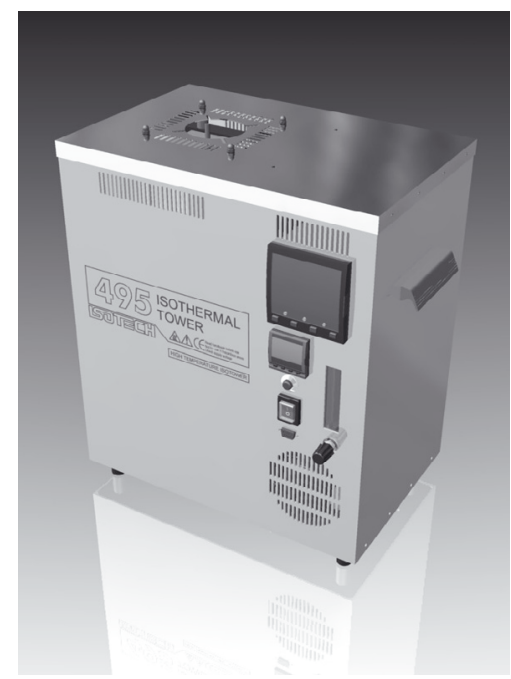

Picture 1. High temperature isothermal tower.

\section{Discussion of silver point results}

The Silver cell was constructed in early June 2009 and the total time at temperature some $200 \mathrm{~h}$, during which it was cycled a number of times through its melt/freeze curves, probably remaining molten for $80 \mathrm{~h}$ of the total test time.

During the whole test sequence the cell was within the metallic heat siphon with $0.2 \mathrm{l} / \mathrm{m}$ of $6 \mathrm{~N} 0$ Argon flux around it.

A small amount of corrosion of the first sacrificial graphite washer was observed.

Regarding the performance of the cell its curves remained consistent with other cells made with this lot of Silver having quartz encapsulation.

Regarding the model $108462 / \mathrm{s}$ thermometer, it remained very stable during the tests including being removed from the cell and reinserted during the cold rod process.

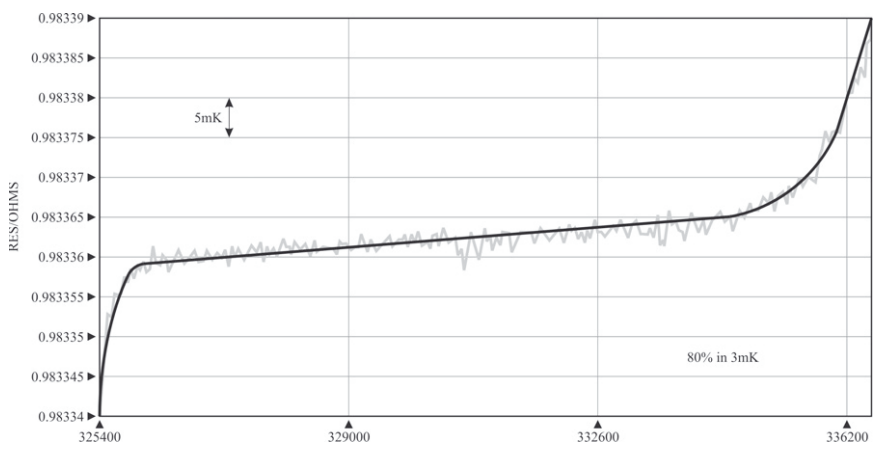

Fig. 2. Silver cell melt curve.

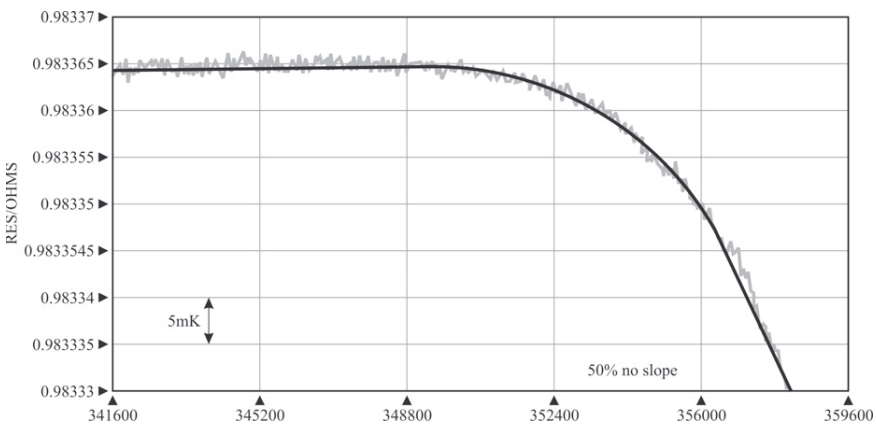

Fig. 3. Silver cell freeze curve.

These results are encouraging and suggest little or no contamination over a $200 \mathrm{~h}$ period of either the cell or its monitor thermometer.

\section{The Copper point}

The same Isotower used for the Silver Point was used. The Silver Cell was removed from the Isotower and replaced by the Copper Cell.

Unlike the Silver Point there are no tried and tested measurement strategies and pre-published results at the Copper Point, however it seems logical to evaluate the Copper Cell by taking it through repeat melts and freezes, noting the slopes and examining the coincidence between melt and freeze, and for this a stable thermometer is required [3-8] (Figs. 4-6).

A new $108462 / \mathrm{S}$ thermometer was used and the results first of all show a drift due to the thermometer as it stabilises. However it rapidly becomes stable enough to enable the melt and freeze slopes to be recorded (Tabs. 1-4).

The Apparatus used was a Wilkins $1 \Omega$ Reference Resistor held at $20^{\circ} \mathrm{C}$, a microK 100 , the new thermometer and an Isotower containing the Copper Cell.

During one freeze the thermometer was withdrawn $40 \mathrm{~mm}$ to test the immersion characteristics of the thermometer, no measurable change occurred (less than $1 \mathrm{mk}$ ).

The Copper Cell had $80 \%$ melt slopes of $5,15,7 \&$ $8 \mathrm{mK}$. The $50 \%$ freeze slopes were $5,0,3$ and $4 \mathrm{mK}$. 


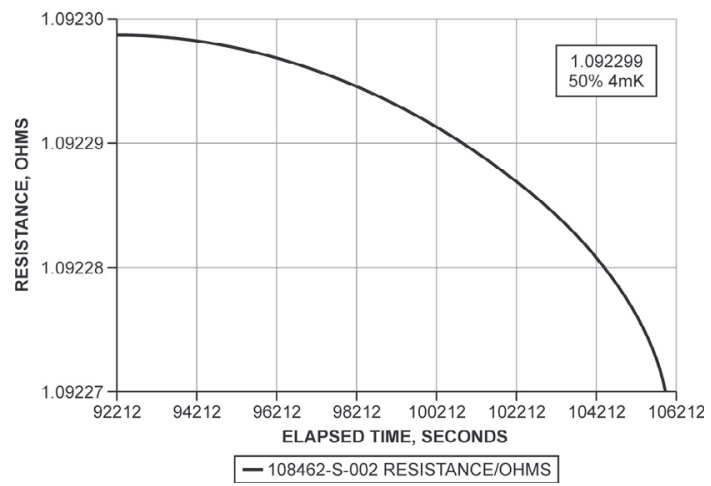

Fig. 4. Siphonic copper cell - freeze plateau - 9th January 2010.

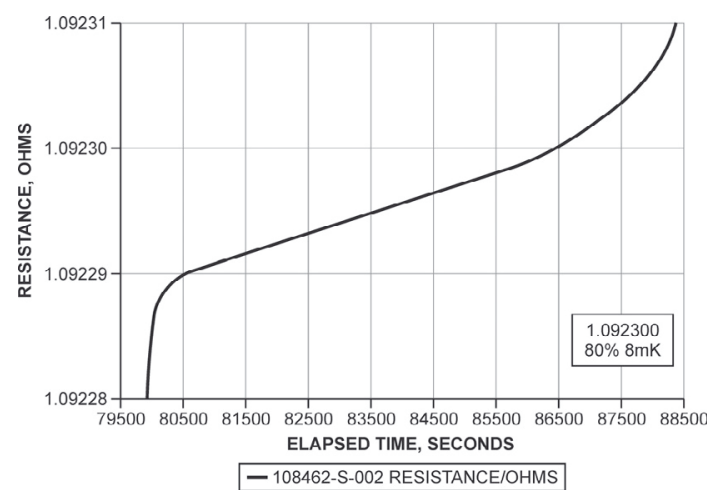

Fig. 5. Siphonic copper cell - melt plateau - 9th January 2010.

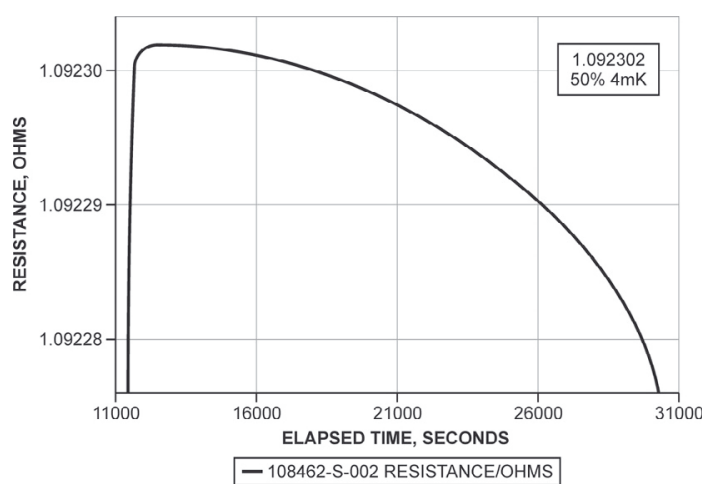

Fig. 6. Siphonic copper cell - freeze plateau - 8th January 2010 .

Coincidence between the liquidus and solidus temperatures was $1 \mathrm{mK}$ for the most stable melt/freeze.

\section{Discussion of the Copper point results}

A simple Combined Cell, Apparatus and Immersion Compensation device has been designed and built for the Silver and Copper point.

It uses techniques adapted from radiation pyrometry to create a metal clad solution without quartz glass.

To measure its melt/freeze characteristics a new thermometer was produced incorporating the major findings
Table 1. First sequence of measurements at the copper point.

\begin{tabular}{lll}
\hline & Slopes & Rcu \\
\hline 3 Hour Melt & $80 \% 5 \mathrm{mK}$ & $1.092,387 \Omega$ \\
9 Hour Freeze & $50 \% 5 \mathrm{mK}$ & $1.092,362 \Omega$ \\
4 Hour Melt & $80 \% 15 \mathrm{mK}$ & $1.092,350 \Omega$ \\
8 Hour Freeze & $50 \%$ Flat & $1.092,308 \Omega$ \\
\hline
\end{tabular}

Table 2. Second sequence of CuPt measurements.

\begin{tabular}{lll}
\hline & Slopes & Rcu \\
\hline 1 Hour Melt & $80 \% 7 \mathrm{mK}$ & $1.092,310 \Omega$ \\
4 Hour Freeze & $50 \% 3 \mathrm{mK}$ & $1.092,302 \Omega$ \\
2 Hour Melt & $80 \% 8 \mathrm{mK}$ & $1.092,300 \Omega$ \\
3 Hour Freeze & $50 \% 4 \mathrm{mK}$ & $1.092,299 \Omega$ \\
\hline
\end{tabular}

Table 3. Third sequence of CuPt measurements.

\begin{tabular}{lll}
\hline & Slopes & Rcu \\
\hline 3 Hour Melt & $80 \% 8 \mathrm{mK}$ & $1.092,298 \Omega$ \\
6 Hour Freeze & $50 \% 4 \mathrm{mK}$ & $1.092,294 \Omega$ \\
\hline
\end{tabular}

Table 4. Fourth sequence of $\mathrm{CuPt}$ measurements.

\begin{tabular}{lll}
\hline & Slopes & Rcu \\
\hline 8 Hour Melt & $80 \% 10 \mathrm{mK}$ & $1.092,293 \Omega$ \\
8 Hour Freeze & $50 \% 5 \mathrm{mK}$ & $1.092,287 \Omega$ \\
\hline
\end{tabular}

of high temperature thermometer researches over the past twenty years.

The resulting thermometer is both robust, stable and functions without contamination up to and including the Copper point.

Concerning the measured slopes, these averaged $9 \mathrm{mK}$ for $80 \%$ melt and $3 \mathrm{mK}$ for $50 \%$ freeze, and included any drift due to the new thermometer's stabilisation.

I have no guide on what to expect from a $6 \mathrm{~N}$ Copper Cell, however if it were a Silver $6 \mathrm{~N}$ Cell I might expect 2 to $4 \mathrm{mK} 80 \%$ melt slope and 1 to $2 \mathrm{mK}$ for $50 \%$ freeze and so the results seem reasonable.

The results do not deteriorate and this suggests that the cell is not contaminating as the melts and freezes progress.

Not mentioned here is oxygen which is a big problem at the Copper Point. One researcher points out that after blanketing Copper with 6N0 Argon the oxygen is reduced to about $3 \mathrm{ppm}$ (which causes $15 \pm 5 \mathrm{mK}$ depression in the Copper Point).

The Copper Cell was therefore taken to $1100{ }^{\circ} \mathrm{C}$ under vacuum for $24 \mathrm{~h}$ and a 5 th sequence of melt and freeze measured (Tab. 5).

The liquidus resistance was identical (within $1 \mathrm{mK}$ ) to the freeze resistance of sequence 4 (Tab. 4) suggesting that the Copper Cell is oxygen free. 
Table 5. Fifth sequence of CuPt measurements.

\begin{tabular}{lll}
\hline & Slopes & Rcu \\
\hline 3 Hour Melt & $80 \% 8 \mathrm{mK}$ & $1.092,287 \Omega$ \\
3 Hour Freeze & $50 \%$ Flat $^{+}$ & $1.092,283 \Omega$ \\
\hline
\end{tabular}

+ Apparatus failed before freeze was complete.

\section{Conclusion}

The Copper point has become easy to use, easily transportable, robust point for Contact Thermometry, with long plateaus.

Its properties can now be measured to milliKelvin sensitivities using easily available bridges and a new thermometer developed specifically for the purpose.

\section{Future work}

Having now obtained a sample of Copper with only $85 \mathrm{ppb}$ impurities, we plan to repeat the melt/freeze process with this new material.

We also plan to incorporate the Copper Point into our UKAS Schedule for contact thermometers.

\section{References}

1. J.P. Tavener, Improvements Relating to the Calibration of Thermometers, NCSLi, 2009

2. Supplementary Information for the ITS-90, p. 67, ISBN, 92-822-2111-3

3. M. Megharfi, Realization of Open Copper Fixed Point Cells for Thermocouple Calibration. BNM-LNE Paris, Temperature, Vol. 7 (2003)

4. N.P. Moiseera, A.I. Pokhodum, Approximation of the ITS90 with High Temperature Thermometers up to $1085^{\circ} \mathrm{C}$, Temperature, Vol. 7 (2003)

5. A.D. McLachlan, The Stability of the Freezing Point of Copper as a Temperature Standard, Temperature - Its Measurement and Control in Science and Industry (The Instrument Society of America, 1972), Vol. 4

6. J.P. Evans, High Temperature Platinum Resistance Thermometry, Temperature - Its Measurement and Control in Science and Industry (The Instrument Society of America, 1972), Vol. 4

7. F. Edler, Intercomparison of Copper Fixed Point Cells by Pt/Pd Thermocouples, Int. J. Thermophys. 29, 171 (2005)

8. F. Righini, A New Determination of the Freezing Point of Copper (IMGC, Italy) 